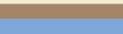

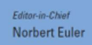

Journal of Nonlinear Mathematical Physics

\title{
Homogeneous Isotropic Turbulence: Geometric and Isometry Properties of the 2-point Velocity Correlation Tensor
}

Vladimir N. Grebenev, Martin Oberlack

To cite this article: Vladimir N. Grebenev, Martin Oberlack (2018) Homogeneous Isotropic Turbulence: Geometric and Isometry Properties of the 2-point Velocity Correlation Tensor, Journal of Nonlinear Mathematical Physics 25:4, 650-672, DOI: https://doi.org/10.1080/14029251.2018.1503447

To link to this article: https://doi.org/10.1080/14029251.2018.1503447

Published online: 04 January 2021 


\title{
REVIEW ARTICLE
}

\section{Homogeneous Isotropic Turbulence: Geometric and Isometry Properties of the 2-point Velocity Correlation Tensor}

\author{
Vladimir N. Grebenev* \\ Institute of Computational Technologies SD RAS, Lavrentjev ave. 6 \\ Novosibirsk, 630090 Russia \\ vngrebenev@gmail.com \\ Martin Oberlack \\ Chair of Fluid Dynamics, Department of Mechanical Engineering, TU Darmstadt, Otto-Berndt-Str. 2 \\ 64287 Darmstadt, Germany \\ oberlack@fdy.tu-darmstadt.de
}

Received 5 March 2018

Accepted 27 April 2018

The emphasis of this review is both the geometric realization of the 2-point velocity correlation tensor field $B_{i j}\left(x, x^{\prime}, t\right)$ and isometries of the correlation space $K^{3}$ equipped with a (pseudo-) Riemannian metrics $d s^{2}(t)$ generated by the tensor field. The special form of this tensor field for homogeneous isotropic turbulence specifies $d s^{2}(t)$ as the semi-reducible pseudo-Riemannian metric. This construction presents the template for the application of methods of Riemannian geometry in turbulence to observe, in particular, the deformation of length scales of turbulent motion localized within a singled out fluid volume of the flow in time. This also allows to use common concepts and technics of Lagrangian mechanics for a Lagrangian system $\left(M^{t}, d s^{2}(t)\right)$, $M^{t} \subset K^{3}$. Here the metric $d s^{2}(t)$, whose components are the correlation functions, evolves due to the von Kármán-Howarth equation. We review the explicit geometric realization of $d s^{2}(t)$ in $K^{3}$ and present symmetries (or isometric motions in $K^{3}$ ) of the metric $d s^{2}(t)$ which coincide with the sliding deformation of a surface arising under the geometric realization of $d s^{2}(t)$. We expose the fine structure of a Lie algebra associated with this symmetry transformation and construct the basis of differential invariants. Minimal generating set of differential invariants is derived. We demonstrate that the well-known Taylor microscale $\lambda_{g}$ is a second-order differential invariant and show how $\lambda_{g}$ can be obtained by the minimal generating set of differential invariants and the operators of invariant differentiation. Finally, we establish that there exists a nontrivial central extension of the infinite-dimensional Lie algebra constructed wherein the central charge is defined by the same bilinear skew-symmetric form $c$ as for the Witt algebra which measures the number of internal degrees of freedom of the system. For turbulence, we give the asymptotic expansion of the transversal correlation function for the geometry generated by a quadratic form.

Keywords: homogeneous isotropic turbulence; two-point correlation tensor; infinite-dimensional Lie algebra; minimal set of differential invariants.

2000 Mathematics Subject Classification: 53B21, 53B30, 76F05

$\overline{{ }^{*} \text { Corresponding author }}$ 


\section{Introduction}

We deal with homogeneous isotropic turbulence and the 2-point velocity correlation tensor field $B_{i j}\left(x^{\prime}, x, t\right)$ where $x^{\prime}$ and $x$ are the points of a three-dimensional space filled by turbulent fluid. The assumption of homogeneity and isotropy of turbulent flow (invariance with respect to rotation, reflection, and translation) implies that this tensor depends only on the length of the correlation vector $\vec{r}$ and time. Moreover $B_{i j}$ is a symmetric tensor field and takes the diagonal form [11]. Therefore $B_{i j}$ is a metric tensor field given on the correlation space $K^{3}$ and $K^{3}$ can be equipped with a Riemannian metric. The form of the components of $B_{i j}$ specifies this metric as the so-called semi-reducible pseudo-Riemannian metric [8]. The components are the correlation functions that evolve due to the von Kármán-Howarth equation [7]. This is conceptually similar to the Ricci flow that acts directly on the metric under consideration. In order to avoid a redundant complexity of the exposition of material, we use only the elementary information about the structure of the two-point velocity correlation tensor of the velocity fluctuations for homogeneous isotropic flows. The modern theory of the properties and structure of second-order (Cartesian) correlation tensors is given in [15]. We only mention that in the course of this development, the authors examined carefully several important misleading or incorrect statements that have remained uncorrected in the literature of the theory of correlation tensors for homogeneous (or isotropic turbulence). Most of these problems arisen because of confusion over the circumstances in which the generating scalar functions can be, or must, pseudoscalar, see for details [15].

The aim of this review is to present both geometric and isometry properties of the 2-point velocity correlation tensor field $B_{i j}\left(x^{\prime}, x, t\right)$ for the case of homogeneous isotropic turbulence which were obtained in [3]-[6] and demonstrates their impact in turbulence. The review is organized as follows. Section 2 contains the results obtained (see [3] for more details) in a compressed form about the geometric realization of the two-point velocity correlation tensor. In Section 3, we present the Lagrangian system generated by $d s^{2}(t)$ and show that it is reduced to a Lagrangian system of the one-degree of freedom for each fixed time $t$ due to the second conservation law obtained. The first integrals of the equations of geodesic curves form the kinematic conservation laws. Section 4 is devoted to symmetries (isometric motions in $K^{3}$ with the structure of a Riemannian manifold) of the metric $d s^{2}(t)$. In fact, the symmetry transformation looks like as a sliding motion of a surface (the geometric realization of the metric) along itself i.e. the form of this surface is invariant under the symmetry transformation. The properties of an infinite-dimensional Lie algebra obtained are discussed. The basis of differential invariants is constructed and a minimal generating set of differential invariants is derived. The well-known Taylor microscale $\lambda_{g}$ is a second-order differential invariant. We show that $\lambda_{g}$ can be obtained by the minimal generating set of differential invariants and the operators of invariant differentiation. In Section 5 we consider the energy-momentum tensor associated with the metric $d s^{2}$ and show that the components $T(z)$ and $\bar{T}(\bar{z})$ of this tensor are holomorphic functions. Then, in the full analogy of Conformal Field Theory (CFT), we expose the asymptotic expansion both for $T(z)$ and $\bar{T}(\bar{z})$ as $z \rightarrow \infty$ and $\bar{z} \rightarrow \infty$. As a consequence of this result, we get the asymptotic expansion of the transversal correlation function $B_{N N}$ for the large values of the correlation distances. The question about the asymptotic expansion of $B_{N N}$ in the physical space $\mathbb{R}^{3}$ with the standard Euclidian metric is still open. To get the link with physics of isotropic turbulence one needs the construction of a family of isomeric embedding into $\mathbb{R}^{3}$. This is conceptually similar to visualization of Ricci flows (see, for an example [16]) but it demands the specification of the properties of the correlation function $B_{L L}$ which cannot be obtained directly from the von 
Kármán-Howarth equation in view of the equation in unclosed form and demands at least a suitable modeling. Here we are not concerned these questions.

\section{Geometric Realization of the Two-Point Velocity Corellation Tensor}

The results of this Section are given in details in [3].

First we recall the elementary information about the structure of the two-point velocitycorrelation tensor of the velocity fluctuations for homogeneous isotropic flows. The modern theory of the properties and structure of second-order (Cartesian) correlation tensors is given in [15].

The statistical description of fluid turbulence employ the Reynolds decomposition to separate the fluid velocity $\vec{u}$ at a point $\vec{x}$ into its mean and fluctuating components as $\vec{u}=\overrightarrow{\vec{u}}+\vec{u}^{\prime}$. Here $\left(^{-}\right)$is the mean velocity, while $\vec{u}$ is the corresponding fluctuating quantity, usually interpreted as representing turbulence. The two-point correlation tensor is defined by

$$
B_{i j}\left(x, x^{\prime}, t\right)=\overline{\left(u_{i}^{\prime}(x, t)\right)\left(u_{j}^{\prime}\left(x^{\prime}, t\right)\right)} .
$$

where $t \in \mathbb{R}_{+}$and $x, x^{\prime}$ are the points of a three-dimensional space filled by turbulent fluid. Rewritten formula (2.1) as

$$
B_{i j}(x, \vec{r}, t)=\overline{\left(u_{i}^{\prime}(x, t)\right)\left(u_{j}^{\prime}(x+\vec{r}, t)\right)},
$$

the vector $\vec{r}$ is determined by the pair $\left(x, x^{\prime}\right)$ where $x$ and $x^{\prime}$ are the starting point and endpoint correspondingly or $\vec{r}=\vec{x}^{\prime}-\vec{x}$. Therefore we will consider an affine space $K^{3}$ with the adjoined vector space of the correlation vectors $\vec{K}^{3} \equiv\left\{\vec{r}=\left(r^{1}, r^{2}, r^{3}\right)\right\}$. The assumption of homogeneity and isotropy of turbulent flow (invariance with respect to rotation, reflection and translation) implies that this tensor depends only on the length of the correlation vector $\vec{r}$ and time $t$ i.e.

$$
B_{i j}(x, \vec{r}, t)=\left[B_{L L}(|\vec{r}|, t)-B_{N N}(|\vec{r}|, t)\right] \frac{r_{i} r_{j}}{|\vec{r}|^{2}}+B_{N N}(|\vec{r}|, t) \delta_{i j}
$$

Moreover for isotropic turbulence, $B_{i j}(|\vec{r}|, t)$ is a symmetric tensor and the correlations $B_{i j}$ can be expressed by using only the longitudinal correlational function $B_{L L}(|\vec{r}|, t)$ and the transversal correlation function $B_{N N}(|\vec{r}|, t)$ [11] i.e. the correlation tensor $B_{i j}$ takes the diagonal form with the components $B_{11}=B_{L L}$ and $B_{22} \equiv B_{33}=B_{N N}$ in a suitable system of the coordinates of the adjoined vector space. Further instead of directly employing the correlation function $B_{L L}$ and $B_{N N}$, we use their normalized representations $f$ and $g$ where $B_{L L}=\overline{u^{\prime 2}(t)} f(|\vec{r}|, t), B_{N N}=\overline{u^{\prime 2}(t)} g(|\vec{r}|, t)$ with the turbulence intensity equals $\overline{u^{\prime 2}(t)}=B_{L L}(0, t)$. Then the corresponding quadratic form (or linear element) takes the form

$$
d l^{2}(t)=\overline{u^{\prime 2}(t)} f(|\vec{r}|, t) d r^{1^{2}}+\overline{u^{\prime 2}(t)} g(|\vec{r}|, t)\left(d r^{2^{2}}+d r^{3^{2}}\right)
$$

where $d l^{2}(t)$ is an indefinite quadratic form in view of the properties $g(|\vec{r}|, t)$, see below. The normalized transversal correlation function $g$ satisfies the relation (taken from the continuity) [2]

$$
g(|\vec{r}|, t)=f(|\vec{r}|, t)+\frac{r}{2} \frac{\partial}{\partial r} f(|\vec{r}|, t) .
$$

The property that $f$ decays faster that $|\vec{r}|^{-2}$ on infinity together with equation (2.3) yields [2]

$$
\int_{0}^{\infty}|\vec{r}| g(|\vec{r}|, t) d|\vec{r}|=0
$$


Hence $g(|\vec{r}|, t)$ is an alternative sign function. Typical forms of experimentally measured functions $f$ and $g$ are given on Figure 1 .

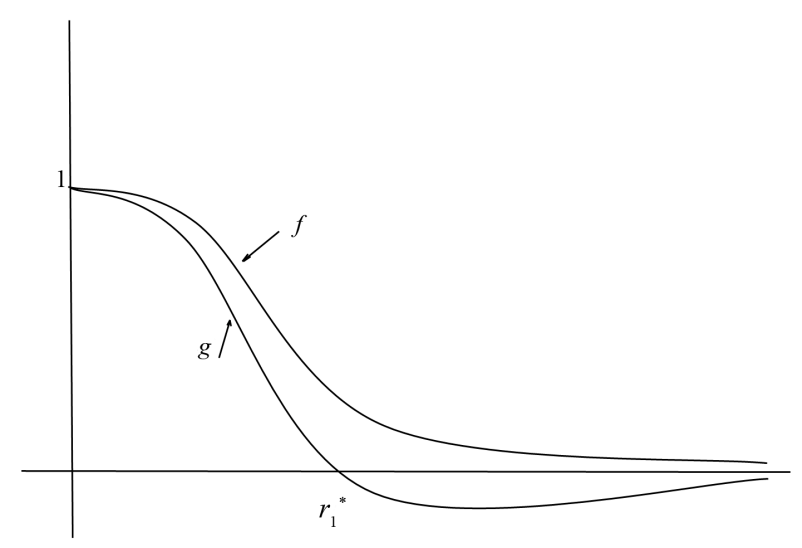

Fig. 1. Typical forms of the normalized longitudinal and transversal correlation functions.

The data presented we use to determine the qualitative behaviors of $f$ and $g$, in particular, the algebraic properties of these correlation functions. Thus, we will assume that $f$ is a positive everywhere function, $g$ changes sign only in interval $\left(-\varepsilon+\left|\vec{r}^{*}\right|,\left|\vec{r}^{*}\right|+\varepsilon\right), \varepsilon>0 . g$ is a positive function on $\left[0, \pm\left|\vec{r}^{*}\right|\right)$ and therefore $g<0$ outside of $\left[-\left|\vec{r}^{*}\right|,\left|\vec{r}^{*}\right|\right]$. The change sign of $g$ means that the quadratic forms $d l^{2}(t)$ have a variable signature. The normalized longitudinal correlational function $f(|\vec{r}|, t)$ is dynamically evolved due to the von Kármán-Howarth equation [9]

$$
\frac{\partial \overline{u^{\prime 2}(t)} f(|r|, t)}{\partial t}=\frac{1}{r^{4}} \frac{\partial}{\partial r} r^{4}\left({\overline{u^{\prime 2}(t)}}^{3 / 2} h(|r|, t)+2 v \frac{\partial}{\partial r} \overline{u^{\prime 2}(t)} f(|r|, t)\right) .
$$

$h$ is the normalized triple-correlation function and $\overline{u^{\prime 2}(t)}$ is the turbulence intensity (a positive everywhere function that vanishes on infinity) or the velocity scale for the turbulent kinetic energy, ${\overline{u^{\prime 2}(t)}}^{3 / 2}$ determines the scale for the turbulence transfer. This single equation directly follows from the Navier-Stokes equation (see, e.g. [11]) and contains two unknowns $f, h$ with the turbulence intensity $\overline{u^{\prime 2}(t)}$ which cannot be defined from (2.5) without the use of additional hypothesis.

If we consider in the correlation space $K^{3}$ an infinite cylindrical domain. Then the metric (induced by the quadratic form $B_{i j}$ ) of the surface which bounds this domain takes the form

$$
d s^{2}(t)=\overline{u^{\prime 2}(t)} f(|\vec{r}|, t) d r^{1^{2}}+\overline{u^{\prime 2}(t)} g(|\vec{r}|, t) \rho^{2} d \phi^{2}
$$

where $\rho$ denotes the Euclidean radius of the cross-section $\{a\} \times S^{1}(\rho)$ of the surface $\mathbb{R} \times S^{1}(\rho)$, $a \in \mathbb{R}$. We can account that $\rho=1$ and identify this manifold with $\mathbb{R} \times S^{1}(1)$. The functions $f, g$ are non-dimensional with $f(0, t)=g(0, t)=1$ and physically $f$ is a positive function such that $f \rightarrow 0$ $(g \rightarrow 0)$ as $|\vec{r}|$ tends to infinite. Moreover, $f$ and $g$ are bounded even functions such that $f \leq 1$, 
$|g| \leq 1$ and $f$ goes faster to zero than $|\vec{r}|^{-2}$, when $|\vec{r}|$ tends to infinity. Physically such behavior of $f$ is acceptable [2] and the map

$$
q(a, t)=\int_{0}^{a} \sqrt{f(|\vec{r}|, t)} d|\vec{r}|, \quad a \in \mathbb{R}
$$

acts as $(-\infty, \infty) \mapsto\left[-\mathbf{L}^{*}(t), \mathbf{L}^{*}(t)\right], \mathbf{L}^{*}(t)=\mathbf{L}(t) / 2 \sqrt{\overline{u^{\prime 2}(t)}}$, where $\mathbf{L}(t)$ is determined by the formulas

$$
\mathbf{L}(t)=2 \int_{0}^{\infty} \sqrt{\overline{u^{\prime 2}(t)} f(|\vec{r}|, t)} d|\vec{r}|
$$

Now we rewrite the metric $d s^{2}(t)$ in the frame of the variable $q$

$$
d s^{2}(t)=\overline{u^{2}(t)}\left\{d q^{2}+G(q, t) d \phi^{2}\right\}, \quad G(q, t)=g(|\vec{r}|, t) .
$$

The metric (2.8) admits an one-parametric group of (isometric) motion $\mathbf{g}_{\tau}(\vec{p}) \equiv \mathbf{g}\left(\vec{p}, a_{1}\right), \vec{p}=$ $(q, \phi)$ of the form

$$
\mathbf{g}_{\tau}:(q, \phi) \mapsto(q, \phi+\chi \tau), \quad \chi=\mathrm{const}
$$

with the generator

$$
X=\xi^{i} \frac{\partial}{\partial p^{i}} \equiv \chi \frac{\partial}{\partial \phi}
$$

The scalar product of the generator $X$ equals

$$
X^{2}=\langle X, X\rangle=\left\langle\chi \frac{\partial}{\partial \phi}, \chi \frac{\partial}{\partial \phi}\right\rangle \equiv \chi^{2}\left\langle\frac{\partial}{\partial \phi}, \frac{\partial}{\partial \phi}\right\rangle=\overline{u^{\prime 2}(t)} \chi^{2} G(q, t)
$$

for each time $t$.

A point $p_{0}$ is called the pole [8] of a (pseudo-)Riemannian manifold $M$ if $p_{0}$ is a fixed point of a group of diffeomorphisms $\mathbf{g}(\vec{x}, \vec{a}), \vec{a}=\left(a_{1}, \ldots, a_{r}\right)$ which acts on $M$.

We note that if $p=p_{0}\left(p_{0}\right.$ is the pole of $\mathbf{g}_{\tau}$ ) then $X^{2}\left(p_{0}\right)=0$ and due to (2.9) $p_{0}$ coincides with the roots of the equation $G(q, \cdot)=0$. Therefore the points $q^{*} \in\left[-\mathbf{L}^{*}(t), \mathbf{L}^{*}(t)\right]$ wherein $G$ vanishes are the poles of $\mathbf{g}_{\tau}$. In view of our assumption on $g(|r|, t)$, the equation $G(q, \cdot)=0$ has only 4 roots $q_{i}^{*}, i=1, \ldots, 4$ such that $\left|q_{1}^{*}\right|=q_{4}^{*}=\mathbf{L}^{*}(t)$ and $\left|q_{2}^{*}\right|=q_{3}^{*}$. Thus the metric (2.8) has the different signature for $q \in I_{1}=\left(q_{2}^{*}, q_{3}^{*}\right)$ and $q \in I_{2}=\left(-\mathbf{L}^{*}(t), q_{2}^{*}\right), q \in I_{3}=\left(q_{3}^{*}, \mathbf{L}^{*}(t)\right)$ respectively where $q_{i}^{*}$ depends on $t$. This metric determines for $q \in I_{1}$ the element of length of the surface of revolution in $\mathbb{R}^{3}$ and the radius-vector $\vec{R}=(q, \phi, \cdot)$ of this surface is given by

$$
\vec{R}(q, \phi, \cdot)=\left(q,\left\{\overline{u^{\prime 2}(t)} G\right\}^{1 / 2}(q, \cdot) \cos \phi,\left\{\overline{u^{\prime 2}(t)} G\right\}^{1 / 2}(q, \cdot) \sin \phi\right) .
$$

Therefore the model manifold defined by (2.8) for $q \in I_{1}$ is a cylindrical-type surface $M_{I_{1}}^{t}=$ $\left(q_{2}^{*}, q_{3}^{*}\right) \times S^{1}(1)$ wherein the radius of the cross-section $\{q\} \times S^{1}(1)$ equals $G^{1 / 2}(q, t)$. For $q \in I_{i}$, 
$i=2,3$ the function $G$ is negative. The metric

$$
d s^{2}(t)=\overline{u^{\prime 2}(t)}\left\{d q^{2}-|G(q, t)| d \phi^{2}\right\}, \quad G(q, t)=g(|\vec{r}|, t)
$$

can be realized (see for details [3]) as a surface of revolution (for each fixed time) in the Minkowski space $\mathbb{R}_{1,2}^{3}$ with the element of length

$$
d \rho^{2}=d x_{1}^{2}-d x_{2}^{2}-d x_{3}^{2}
$$

when the form $d s^{2}(t)$ is of a fixed sign. Here the rotation presents the motion along the pseudocircle of the radius $|G(q, \cdot)|^{1 / 2}, q \in I_{i}$. Indeed, let us fix the point $p_{a}=\left(q_{a}, \phi_{a}\right)$ on the cross-section $\left\{q_{a}\right\} \times S^{1}(1)$ and consider the action of the group $\mathbf{g}_{\tau}$ on $p_{a}$ i.e. the orbit $\mathbf{O}_{p_{a}}: \tau \mapsto \mathbf{g}_{\tau}\left(p_{a}\right)$. This action is a motion along $\left\{q_{a}\right\} \times S^{1}(1)$ and if $p_{a}$ does not coincide with the poles $\mathbf{g}_{\tau}$ then $\mathbf{O}_{p_{a}}$ is a not compact set [8]. In particular, $\mathbf{O}_{p_{a}} \subseteq\left\{\left(x_{1}, x_{2}\right): x_{1}^{2}-x_{2}^{2}=\overline{u^{\prime 2}(t)}\left|G\left(q_{a}, t\right)\right|\right\}$ for each fixed time $t$ which coincides with the so-called pseudo-circle under the embedding $M_{I_{3}}^{t}\left(M_{I_{2}}^{t}\right)$ into the Minkowski space $\mathbb{R}_{1,2}^{3}$. Moreover, the poles are saddle points of a negative index for the orbits $\mathbf{O}_{p}, p \in M_{I_{3}}^{t}\left(M_{I_{2}}^{t}\right)$. The cross-sections $\left\{q_{a}\right\} \times S^{1}(1)$ of $M_{I_{3}}^{t}\left(M_{I_{2}}^{t}\right)$ for $q_{a} \in\left\{q_{3}^{*}, \mathbf{L}^{*}(t)\right\}$ (respectively $\left.q_{a} \in\left\{-\mathbf{L}^{*}(t), q_{2}^{*}\right\}\right)$ are the pseudo-circles of zero radius and consist of the isotropic rays with the initial points $q_{3}^{*}$ and $\mathbf{L}^{*}(t)$ (respectively $-\mathbf{L}^{*}(t)$ and $q_{2}^{*}$ ). The action of $\mathbf{g}_{\tau}$ on the point $p$ is a motion along these piecewise linear isotropic curves when $p \in\left\{-\mathbf{L}^{*}(t), q_{2}^{*}, q_{3}^{*}, \mathbf{L}^{*}(t)\right\}$. We can identify $M_{I_{3}}^{t}\left(M_{I_{2}}^{t}\right)$ with the foliation space of orbits $M_{I_{j}}^{t}=\bigcup_{p} \mathbf{O}_{p}$ and associate the modulus of the transversal correlation function $G(q, t)$ with the length of the velocity vector $\vec{\xi}(p)$ of the orbit $\mathbf{O}_{p}$ by $|\vec{\xi}(p)|=\overline{u^{\prime 2}(t)} \chi \sqrt{|G(q, t)|}$. The length of displacement of the point $p$ (or the length of arch), with respect to the vector field generated by $\mathbf{g}_{\tau}(p)$, is determined by the formula

$$
\lambda\left(\phi_{a}, \phi_{b}\right)=\int_{\phi_{a}}^{\phi_{b}} \chi \sqrt{\overline{u^{\prime 2}(t)} \mid G(q, t)}\left|d \phi \equiv \chi \sqrt{\overline{u^{\prime 2}(t)} \mid G\left(q, t_{c}\right)}\right|\left(\phi_{b}-\phi_{a}\right), \quad \chi=\mathrm{const}
$$

for each fixed time that defines the following length scale along the orbit $\mathbf{O}_{p}$

$$
\lambda_{\mathbf{o}_{p}}=\chi \sqrt{\overline{u^{\prime 2}(t)} \mid G(q, t)} \mid 2 \pi \phi, \quad q \in\left(q_{3}^{*}, \mathbf{L}^{*}(t)\right), \quad \chi=1 .
$$

The constant $\chi$ can be fixed by normalizing the velocity vector $\vec{\xi}(p)$.

The Gaussian curvature $K_{++}$of the manifold $M_{I_{1}}^{t}$ admits a singular behavior at the poles $q_{2}^{*}$ and $q_{3}^{*}$ where $G$ vanishes for $G_{q}\left(q_{i}^{*}, t\right) \neq 0, i=2,3$. In the case when the poles are multiplicative zeroes of some finite order i.e. $G\left(q_{i}^{*}, t\right)=G_{q}\left(q_{i}^{*}, t\right)=\ldots G_{q \ldots q}\left(q_{i}^{*}, t_{c}\right)=0$ then the direct calculations show that again $K_{++}$is a singular function at $q_{i}^{*}$ for all $t$. If zero is of infinite order then $G(q, t) \equiv 0$ in a neighborhood of $q_{2}^{*}\left(q_{3}^{*}\right)$ under the assumption that $G$ is an analytical function. The same argument we can apply to investigation of the behavior of the Gaussian curvature of the manifold $M_{I_{3}}^{t}\left(M_{I_{2}}^{t}\right)$ for the poles $g_{3}^{*}\left(g_{2}^{*}\right)$. In the case of the pole $q_{4}^{*}=\mathbf{L}^{*}(t)\left(q_{1}^{*}=-\mathbf{L}^{*}(t)\right)$ we use that $f$ and therefore $g$ have to go faster to zero than $|\vec{r}|^{-2}$ when $|\vec{r}| \rightarrow \infty$. Employing the formula $G_{q}=f^{-1 / 2} g_{r}, r=|\vec{r}|$ and the above-mentioned assumption about the behavior $f$ (and $G$ ) as $|\vec{r}| \rightarrow \infty$ (and $q \rightarrow \pm \mathbf{L}^{*}(t)$ ), we derive in terms of the variable $|\vec{r}|$ that $|K|$ have to go faster to infinity than $|\vec{r}|^{2}$ when $|\vec{r}| \rightarrow \infty$ $\left(q \rightarrow \pm \mathbf{L}^{*}(t)\right)$. 
We indicate a connection between the Gaussian curvature $K_{++}$of $M_{I_{1}}^{t}$ and the transverse Taylor microscale $\lambda_{g}$ (see, e.g. [11]) which is defined by $\lambda_{g}^{2}(t)=-2\left(g_{r r}(0, t)\right)^{-1}$. It is easy to show that

$$
K_{++}=\frac{1}{\lambda_{g(t)}^{2}}=2\left(\frac{1}{\lambda_{f(t)}^{2}}\right) \quad \text { for the cross-section }\{0\} \times S^{1},
$$

where $\lambda_{f(t)}^{2}=2 \lambda_{g(t)}^{2}$ is the Taylor longitudinal scale. This formula shows that the Gaussian curvature of $M_{I_{1}}^{t}$ is positive for small values of $|q|$. Moreover, the formula (2.11) gives the connection between the geometry of $M_{I_{1}}^{t}$ and the microscales arising in turbulence. Using the well-known relationship between turbulent length scales (see, e.g. [11])

$$
\lambda_{g(t)}=\sqrt{10} \eta^{2 / 3} \ell_{t}^{1 / 3}
$$

where $\eta$ is the Kolmogorov length scale and $\ell_{t}$ is the integral length scale characterizing the large eddies, we can write that

$$
K_{++}=\frac{1}{10 \eta^{4 / 3} \ell_{t}^{2 / 3}} \quad \text { for the cross-section }\{0\} \times S^{1} .
$$

The Kolmogorov scale $\eta$ varies with the viscosity $v$ and the dissipation of turbulent energy $\varepsilon$ according to $\eta=\left(v^{3} / \varepsilon\right)^{1 / 4}$. In the limit of infinite Reynolds numbers or vanishing the viscosity $v, v$ decreases and the Gaussian curvature $K_{++}$restricted on the cross-section $\{0\} \times S^{1}$ grows infinitely. It means that $M_{I_{1}}^{t}$ has singular points at $q=0$ which forms the so-called break circle where the manifold loses smoothness.

The peculiarity of the metric presented consists in arising the singularity of "the shrinking cylinder type" due to alternative sign of the transversal correlation function $B_{N N}$. It means that we can describe "shrinking phenomenon" for a singled out cylinder in terms of singularity points of the metric (2.8).

\section{Lagrangian system}

We write the metric $d s^{2}(t)$ in the conformal form at first for $q \in I_{1}$. Let us consider on the interval $I_{1}$ a new measure $d \zeta$ with the density $\sigma(q, t)=1 / \sqrt{\overline{u^{\prime 2}(t)} G(q, t)}$ for each fixed time and rewrite $d s^{2}(t)$ as

$$
d s^{2}(t)=F(\zeta, t)\left(d \zeta^{2}+d \phi^{2}\right)
$$

where $F(\zeta, t)=\overline{u^{\prime 2}(t)} G(q, t)$. For $q \in I_{i}, i=2,3$ this metric takes the form

$$
d s^{2}(t)=F(\zeta, t)\left(d \zeta^{2}-d \phi^{2}\right)
$$

Here $F(\zeta, t)=\overline{u^{\prime 2}(t)}|G(q, t)|$ and $d \zeta=\sqrt{\overline{u^{\prime 2}(t)} \mid G(q, t)} \mid d q$. The equations for geodesic curves on the manifold $M_{I_{1}}^{t}$ in the frame of (3.1) read

$$
\frac{d^{2} \zeta}{d \theta^{2}}+\frac{F_{\zeta}}{2 F}\left[\left(\frac{d \zeta}{d \theta}\right)^{2}+\left(\frac{d \phi}{d \theta}\right)^{2}\right]=0, \quad \frac{d^{2} \phi}{d \theta^{2}}+\frac{F_{\zeta}}{F} \frac{d \zeta}{d \theta} \frac{d \phi}{d \theta}=0
$$


While for the metric (3.2) given on $M_{I_{i}}^{t}, i=2,3$ we have

$$
\frac{d^{2} \zeta}{d \theta^{2}}+\frac{F_{\zeta}}{2 F}\left[\left(\frac{d \zeta}{d \theta}\right)^{2}-\left(\frac{d \phi}{d \theta}\right)^{2}\right]=0, \quad \frac{d^{2} \phi}{d \theta^{2}}+\frac{F_{\zeta}}{F} \frac{d \zeta}{d \theta} \frac{d \phi}{d \theta}=0
$$

Here we use the coordinates $(\zeta, \theta)$ for each chart of the manifold $M^{t}$. Integrability of these systems of equations is established by the usual way. The systems of equations (3.3) and (3.4) admit the following first integrals

$$
F(\zeta, t) \phi_{\theta}=\mathbf{M}, \quad F(\zeta, t)\left(\zeta_{\theta}^{2}+\phi_{\theta}^{2}\right)=\mathbf{N}
$$

and

$$
-F(\zeta, t) \phi_{\theta}=\mathbf{M}, \quad F(\zeta, t)\left(\zeta_{\theta}^{2}-\phi_{\theta}^{2}\right)=-\mathbf{N}
$$

correspondingly where $\mathbf{M}$ and $\mathbf{N}$ depend on the time $t$ in general. Let us consider now the vector $\vec{M}=F(\zeta, t) \phi_{\theta}[d \zeta, d \phi]_{d l^{2}}$. This is the so-called momentum vector from the cotangent vector space to the one-parametric group of (isometric) motion $\mathbf{g}_{\tau}$. Here $[\cdot, \cdot]_{d l^{2}}$ denotes the vector product with respect to the metric $d \zeta^{2} \pm d \phi^{2}$. The equality $F\left(\zeta, t_{c}\right) \phi_{\theta} \equiv \mathbf{M}=$ const means that the momentum vector $\vec{M}$ is a conserved quantity: $d \vec{M} / d \theta=0$ for each fixed time $t$. On the plane $(d \zeta, d \phi)$ the momentum vector $\vec{M}$ is calculated by the formula $\vec{M}=\left[\vec{p}, \vec{p}_{\theta}\right]_{d l^{2}}, \vec{p}(\theta, t)=(\zeta(\theta, t), \phi(\theta, t))$. Without loss of generality (in view of the invariance of $d s^{2}(t)$ with respect to $\mathbf{g}_{\tau}$ ), we can write $\vec{p}(\theta, t)=F^{1 / 2}(\zeta, t) d \zeta$ and $d \vec{p} / d \theta=\left(F^{1 / 2}\right)_{\theta} d \zeta+F^{1 / 2} d \zeta_{\theta}=\left(F^{1 / 2}\right)_{\theta} d \zeta+F^{1 / 2} \phi_{\theta} d \phi$. Therefore

$$
\vec{M}=\left[\vec{p},\left(F^{1 / 2}\right)_{\theta} d \zeta\right]_{d l^{2}}+\left[\vec{p}, F^{1 / 2} \phi_{\theta} d \phi\right]_{d l^{2}}=F \phi_{\theta}[d \zeta, d \phi]_{d l^{2}}
$$

where the first bracket vanishes and the length of the vector $[d \zeta, d \phi]_{d l^{2}}$ equals -1 (1) for the signature $(+-)((++))$. Using the above-mentioned first integrals or the compatible differential constraints to (3.3), (3.4), the reduction of (3.3) on invariant manifolds defined by these constraints leads to the following equation

$$
\frac{d^{2} \zeta}{d \theta^{2}}+\frac{F_{\zeta}}{2 F}\left(\frac{ \pm \mathbf{N} \mp 2 \mathbf{M}^{2} F^{-1}}{F}\right)=0
$$

where the upper sign in $\pm \cdots \mp$ corresponds to the signature $(++)$ and the lower symbols are reserved for the signature $(+-)$. The second equation of this system is satisfied identically on the constraint $\pm F(\zeta, t) \phi_{\theta}=\mathbf{M}$ for the different signature. As a result, we obtain

$$
\frac{d^{2} \zeta}{d \theta^{2}}=-\frac{d V}{d \zeta}, \quad \text { where } V=\mp \frac{\mathbf{N}}{2 F(\zeta, t)} \pm \frac{\mathbf{M}^{2}}{2 F^{2}(\zeta, t)}+\text { const }
$$

considering $t$ as a parameter. Equation (3.5) coincides with the well-known equation of the motion of a unit mass point in the potential field with the effective potential energy $V$ (the terminology of Newtonian mechanics is used). Then the Lagrangian $\mathbf{S}_{M_{I_{i}}^{t}}$ and the "total mechanical" energy $E_{M_{I_{i}}^{t}}$ 
read

$$
\mathbf{S}_{M_{I_{i}}^{t}}=\frac{1}{2}\left(\frac{d \zeta}{d \theta}\right)^{2}-V, \quad E_{M_{I_{i}}^{t}}=\frac{1}{2}\left(\frac{d \zeta}{d \theta}\right)^{2}+V .
$$

Here $E_{M_{I_{i}}^{t}}$ is the conserved quantity on solutions of equation (3.5) for each fixed time $t$. Therefore the couple $\left(M_{I_{i}}^{t}, d s^{2}(t)\right)$ generates a Lagrangian system of the one-degree of freedom. Hence

$$
d \theta=\frac{d \zeta}{\sqrt{2\left(E_{M_{I_{i}}^{t}}-V\right)}}, \quad d \phi= \pm \frac{\mathbf{M} d \theta}{F(\zeta, t)}
$$

and the standard excepting $d \theta$ leads to the following equation for $\zeta=\zeta(\phi)$

$$
\frac{d \zeta}{d \phi}=\frac{F\left(\zeta, t_{c}\right)}{ \pm \mathbf{M}} \sqrt{2\left(E_{M_{I_{i}}^{t}}-V\right)} .
$$

Geometrically, the parameter $\theta$ is the length of arch of a non-isotropic geodesic curve with the initial position at the corresponding pole. If this length is changed then in the course of during this process the "total mechanical" energy and momentum are preserved quantities along geodesic curves. The angle $\alpha$ between the vector

$$
\vec{\xi}(p)=\left.\frac{d \mathbf{g}_{\tau}(p)}{d \tau}\right|_{\tau=0} \text { and the velocity vector } \vec{v}_{\vec{\gamma}}=\left(\zeta_{\theta}, \phi_{\theta}\right)
$$

of the geodesic curve $\vec{\gamma}$ equals

$$
\cos \alpha=\frac{\left\langle\vec{v}_{\vec{\gamma}}, \vec{\xi}(p)\right\rangle_{d s^{2}}}{\sqrt{\left|\left\langle\vec{v}_{\vec{\gamma}}, \vec{v}_{\vec{\gamma}}\right\rangle_{d s^{2}}\langle\vec{\xi}(p), \vec{\xi}(p)\rangle_{d s^{2}}\right|}}=\frac{\phi_{\theta} F}{\sqrt{\left|F\left(\zeta_{\theta}^{2} \pm \phi_{\theta}^{2}\right)\right|}}=\frac{ \pm \mathbf{M}}{\sqrt{| \pm \mathbf{N} F|}}
$$

or

$$
\sqrt{F} \cos \alpha= \pm \mathbf{M} / \sqrt{| \pm \mathbf{N}|} .
$$

The case of $\cos \alpha=0$ corresponds to the motion along the orbit $\mathbf{O}_{p}$, where $\vec{\xi}^{2}\left(\mathbf{g}_{\tau}(p)\right)=$ const ( $F \equiv$ const) and this orbit presents unclosed curve in the case of $\left(M_{I_{i}}^{t}, d s^{2}(t)\right)$ for $i=2,3$. Notice that in the sharp contrast to the previous consideration about the dynamics of a fluid particle on the surface of revolution $M_{I_{1}}^{t}$ the zero value of $\left|\vec{\xi}^{2}\left(\mathbf{g}_{\tau}(p)\right)\right|$ means that $\mathbf{O}_{p}$ belongs to the so-called isotropic curve $\left(d s^{2}(t)=0\right)$ which is given by $\phi=\zeta-\zeta_{0}\left(\right.$ or $\left.\phi=-\left(\zeta-\zeta_{0}\right)\right)$. This curve coincides with the pseudo-circle of zero radius. The canonical parameter $\theta$ (the length of the corresponding isotropic curve) is defined by the formula

$$
\theta=\int_{\zeta_{0}}^{\zeta} F(\zeta, t) d \zeta \quad \text { or in the variable } q \quad \theta=\int_{q_{0}}^{q} \sqrt{\overline{u^{\prime 2}(t)}|G(q, t)|} d q
$$

Here $\left(\zeta_{0}, 0\right)$ is the coordinate of the corresponding pole of $M_{I_{3}}^{t_{c}}\left(M_{I_{2}}^{t}\right)$. The existence of closed geodesic curves depends on the form of the potential $V$.

The above-mentioned conservation laws are standard consequence of invariance of the flow with respect to the translation and rotation groups. Note that the quantity $F(\zeta, t)\left(\zeta_{\theta}^{2} \pm \phi_{\theta}^{2}\right)$ means geometrically that along geodesic curves the length of tangent vectors is conserved. Together with the length of the momentum vector $\vec{M}$ they present new conserved quantities arising in homogeneous isotropic turbulence, see [4]. 


\section{Isometries}

We consider the functional of simple action in $\left(M^{t}, d s^{2}\right)$ and present isometric motions in $K^{3}$. The peculiarity of these transformations consists in: we look for their in the class of equivalence transformations that preserve the length of tangent vectors $F(\zeta, t)\left(\zeta_{\theta}^{2} \pm \phi_{\theta}^{2}\right)$ along geodesic curves with fixed endpoints. For more details see [5].

\subsection{Equivalence transformation}

Instead of the variables $(\rho, \phi)$, we consider

$$
\xi=e^{\zeta} \cos \phi, \quad \eta=e^{\zeta} \sin \phi
$$

then

$$
d s^{2}=\Lambda^{2}(\rho, t)\left(d \xi^{2}+d \eta^{2}\right)
$$

and

$$
d s^{2}=\Lambda^{2}(\rho, t)\left(d \xi^{2}-d \eta^{2}\right), \quad \Lambda^{2}(\rho, t) \equiv F(\zeta, t)
$$

for

$$
\xi=e^{\zeta} \cosh \phi, \quad \eta=e^{\zeta} \sinh \phi .
$$

Consider the set of piecewise smooth curves $\Omega\left(M_{I_{i}}^{t}, v, w\right): \gamma_{I_{i}}: J \rightarrow M_{I_{i}}^{t}$ with fixed endpoints $\gamma_{I_{i}}(0)=$ $v_{i}$ and $\gamma_{I_{i}}(1)=w_{i}$. Let $L_{\gamma_{I_{i}}}^{t}: \Omega\left(M_{I_{i}}^{t}, v_{i}, w_{i}\right) \rightarrow \mathbb{R}$ be the functional of length (the simple action) for each fixed time $t$. Then the formula

$$
d_{I_{i}}^{t}(v, w)=\min _{\gamma_{i} \in \Omega\left(M_{I_{i}}^{t}, v_{i}, w_{i}\right)} L_{\gamma_{i}}^{t}(\gamma), \quad L_{\gamma_{l_{i}}}^{t}=\int_{\gamma_{l_{i}}} \sqrt{\Lambda^{2}(\rho, t)\left(\xi_{\tau}^{2}+\eta_{\tau}^{2}\right)} d \tau
$$

defines the function of distance $d_{I_{i}}^{t}: M_{I_{i}}^{t} \times M_{I_{i}}^{t} \rightarrow \mathbb{R}$. We can account that $\tau$ is the so-called natural parameter i.e

$$
\Lambda^{2}(\rho, t)\left(\xi_{\tau}^{2} \pm \eta_{\tau}^{2}\right)=1 \quad \text { along the curve } \gamma_{I_{i}} .
$$

Therefore

$$
L_{\gamma_{l_{i}}}^{t}=\int_{\gamma_{l_{i}}} 1 \cdot d \tau=\tau_{\gamma_{i}}
$$

where the symbol $\tau_{\gamma_{i}}$ denotes the length of $\gamma_{I_{i}}$. Let $\vec{r}_{i}=\vec{r}_{i}(\xi, \eta)$ be a local realization of $M_{I_{i}}^{t}$ in a chart $(\xi, \eta)$. Consider an infinitesimal transformation of the variables $\xi$ and $\eta$

$$
\xi^{*}=\xi+\delta \xi, \quad \eta^{*}=\eta+\delta \eta,
$$

assuming that $\vec{r}_{i}$ is the differentiable vector-function. Then we can write

$$
\vec{r}_{i}\left(\xi^{*}, \eta^{*}\right)=\vec{r}_{i}(\xi, \eta)+\mathbf{X} \delta \xi+\mathbf{Y} \delta \eta
$$

or

$$
\vec{r}_{i}^{*}(\xi, \eta)=\vec{r}_{i}(\xi, \eta)+\vec{U}(\xi, \eta)
$$

where $\vec{U}(\xi, \eta)$ denotes an infinitesimal deformation of the surface with the radius-vector $\vec{r}_{i}(\xi, \eta)$. We can account that $\vec{r}_{i}^{*}(\xi, \eta)$ is the radius-vector of the deformed surface and $\gamma_{I_{i}}$ is transformed into 
$\gamma_{I_{i}}^{*}$ which belongs to the same class of functions just as $\gamma_{I_{i}}$. In order to investigate the invariance of $d_{I_{i}}^{t}$ under the action of infinitesimal transformations, it is sufficient to prove that the length of arch of extremals of the functional above is invariant. Instead of the tangent vector $(\xi, \eta)$, we consider the co-vector $\left(\tau_{\xi}, \tau_{\eta}\right)$ defined by the formulas (for brevity we omitted the index $\gamma_{I_{i}}$ for $\tau$ )

$$
\xi_{\tau}=\frac{\tau_{\xi}}{\Lambda^{2}}, \quad \eta_{\tau}=\frac{\tau_{\eta}}{\Lambda^{2}}
$$

Then (4.1) is transformed into

$$
\left.\tau_{\xi}^{2}+\tau_{\eta}^{2}=\Lambda^{2}(\rho, t), \quad \tau_{\xi}^{2}-\tau_{\eta}^{2}=\Lambda^{2}(\rho, t)\right)
$$

This equation is the eikonal-type one (the relativistic eikonal equation for the signature $(+-)$ ). Therefore in order to find isometries of $L_{\gamma I_{i}}^{t}$ we can consider symmetries admitted by equation (4.5) that leaves $\tau$ invariant. The restatement of the variational symmetry in the terms of symmetry of partial differential equations (4.5) enables us to extend the class of isometry transformations admitted by the functional $L_{\gamma I_{i}}^{t}$. More exactly, we extend the variational symmetry up to the equivalence transformations. Recall that equivalence transformations for a differential equation in given class is a change of variables which maps the equation to another equation in the same class. An equivalence transformation admitted by (4.5) is a point transformation given on $\left(\xi, \eta, u^{1}, u^{2}\right)$ space where $u^{1}=\tau, u^{2}=\Lambda^{2}$. Infinitesimally, we look for an operator in the following form [12], [13]

$$
Y=\mu\left(\xi, \eta, u^{1}, u^{2}\right) \frac{\partial}{\partial \xi}+v\left(\xi, \eta, u^{1}, u^{2}\right) \frac{\partial}{\partial \eta}+\lambda^{i}\left(\xi, \eta, u^{1}, u^{2}\right) \frac{\partial}{\partial u^{i}}
$$

where the coefficients are defined due to the equation

$$
\left.Y_{1}\right|_{(4.5)}=0
$$

Here $Y_{1}$ denotes the first prolongation of $Y$. In the case of the signature $(++)$ the coefficients of the operator $Y$ have been calculated in [12]. For the relativistic eikonal equation i.e. for the signature $(+-)$ these calculations are given in [5]. The infinitesimal operator $Y$ reads:

$$
Y=\Phi(\xi, \eta) \frac{\partial}{\partial \xi}+\Psi(\xi, \eta) \frac{\partial}{\partial \eta}+\gamma(\tau) \frac{\partial}{\partial \tau}+2\left(\frac{d \gamma}{d \tau}-\Phi_{\xi}(\xi, \eta)\right) \Lambda^{2} \frac{\partial}{\partial \Lambda^{2}}
$$

Its Lie (infinite-dimensional) subalgebra is of the form

$$
X=\Phi(\xi, \eta) \frac{\partial}{\partial \xi}+\Psi(\xi, \eta) \frac{\partial}{\partial \eta}-2 \Phi_{\xi}(\xi, \eta) \Lambda^{2} \frac{\partial}{\partial \Lambda^{2}}
$$

and $\tau$ is a scalar invariant of $X$. Therefore $X$ is a symmetry operator admitted by the functional $L_{\gamma_{l_{i}}}^{t}$. Here the functions $\Phi(\xi, \eta)$ and $\Psi(\xi, \eta)$ satisfy the Cauchy-Riemann differential equations $\Phi_{\xi}=\Psi_{\eta}$ and $\Phi_{\eta}=-\Psi_{\xi}$ in the case of the eikonal equation and $\Phi_{\xi}=\Psi_{\eta}, \Phi_{\eta}=\Psi_{\xi}$ (the so-called $h$-conjugate functions [10]) for the relativistic eikonal equation. To expose a fine structure of the equivalence transformation generated by $X$, we consider the case of the signature $(++)$ and the 
complex coordinates $z=\xi+i \eta$ and $\bar{z}=\xi-i \eta$. Then

$$
d s^{2}=\Lambda^{2}(z \bar{z}) d z d \bar{z}
$$

or more exactly

$$
d s^{2}=\frac{\Lambda^{2}(z \bar{z})}{2}(d z \otimes d \bar{z}+d \bar{z} \otimes d z)
$$

The operator $X$ takes the form

$$
X_{1}=F(z) \frac{d}{d z}+\bar{F}(\bar{z}) \frac{d}{d \bar{z}}-F_{z}(z) \Lambda^{2}(z \bar{z}) \frac{d}{\partial \Lambda^{2}}-\bar{F}_{\bar{z}}(\bar{z}) \Lambda^{2}(z \bar{z}) \frac{d}{\partial \Lambda^{2}} .
$$

Here $F=\Phi+i \Psi, \bar{F}=\Phi-i \Psi$ and we use that $F(z, \bar{z}) \equiv F(z), \bar{F}(z, \bar{z}) \equiv \bar{F}(\bar{z})$ for the holomorphic function $F$. The tangent space is spanned by

$$
\begin{aligned}
& \frac{d}{d z}=\frac{1}{2}\left(\frac{\partial}{\partial \xi}-i \frac{\partial}{\partial \eta}\right), \\
& \frac{d}{d \bar{z}}=\frac{1}{2}\left(\frac{\partial}{\partial \xi}+i \frac{\partial}{\partial \eta}\right) .
\end{aligned}
$$

For small perturbations $z \mapsto z+\varepsilon(z)$ and $\bar{z} \mapsto \bar{z}+\bar{\zeta}(\bar{z})$ we find the representation of the operator $X_{1}$. Infinitesimal holomorphic transformations of the variables $z$ and $\bar{z}$ read

$$
z^{*}=z+\varepsilon(z)=z+F(z) \delta s, \quad \bar{z}^{*}=\bar{z}+\bar{\varepsilon}(\bar{z})=\bar{z}+\bar{F}(\bar{z}) \delta s
$$

which are generated by the vector field

$$
\left(F(z) \frac{d}{d z}, \bar{F}(\bar{z}) \frac{d}{d \bar{z}}\right)
$$

Using the Laurent series

$$
\varepsilon(z)=-\sum_{n=-\infty}^{\infty} \varepsilon_{n} z^{n+1}, \quad \bar{\varepsilon}(\bar{z})=-\sum_{n=-\infty}^{\infty} \bar{\varepsilon}_{n} \bar{z}^{n+1}
$$

where $\varepsilon_{n}\left(\bar{\varepsilon}_{n} \bar{z}\right)$ are infinitesimal small numbers. We can look at $\varepsilon_{n} z^{n+1}, \bar{\varepsilon}_{n} \bar{z}^{n+1}$ as the harmonics of decomposition of $\zeta(z), \bar{\zeta}(\bar{z})$ with respect to the basis functions $\left\{z^{n+1}\right\}\left(\left\{\bar{z}^{n+1}\right\}\right)$. Each harmonics generate the transformations $z \rightarrow z^{\prime} \equiv z-\varepsilon_{n} z^{n+1}, \bar{z} \rightarrow \bar{z}^{\prime} \equiv \bar{z}-\bar{\varepsilon}_{n} \bar{z}^{n+1}$ and the corresponding infinitesimal generators:

$$
l_{n}=-z^{n+1} \frac{d}{d z}, \quad \bar{l}_{n}=-\bar{z}^{n+1} \frac{d}{d \bar{z}},
$$

where $\varepsilon_{n}, \bar{\varepsilon}_{n}$ are the parameters of the transformation, presents the basis of the infinite dimensional conformal Lie algebra (two copies of the Witt algebra)

$$
Z=F(z) \frac{d}{d z}+\bar{F}(\bar{z}) \frac{d}{d \bar{z}} .
$$

Therefore the basis of the operator $X_{1}$

$$
X_{1}=F(z) \frac{d}{d z}+\bar{F}(\bar{z}) \frac{d}{d \bar{z}}-F_{z}(z) \Lambda^{2}(z \bar{z}) \frac{d}{d \Lambda^{2}}-\bar{F}_{\bar{z}}(\bar{z}) \Lambda^{2}(z \bar{z}) \frac{d}{d \Lambda^{2}}
$$


is $\left\{k_{n}, \bar{k}_{n}\right\}, n \in \mathbb{Z}$

$$
k_{n}=-z^{n+1} \frac{d}{d z}-(n+1) z^{n} \Lambda^{2} \frac{d}{d \Lambda^{2}}, \quad \bar{k}_{n}=-\bar{z}^{n+1} \frac{d}{d \bar{z}}-(n+1) \bar{z}^{n} \Lambda^{2} \frac{d}{d \Lambda^{2}} .
$$

The factor $\Lambda^{2}$ is transformed into

$$
\Lambda^{2^{*}}=\frac{\Lambda^{2}}{z_{z}^{*}}, \quad \text { and } \Lambda^{2^{* *}}=\frac{\Lambda^{2^{*}}}{\bar{z}_{\bar{z}}^{*}}
$$

under the change of variables $z \mapsto z^{*}$ and $\bar{z} \mapsto \bar{z}^{*}$ correspondingly.

The linear hull of the direct sum $k_{n} \oplus \bar{k}_{n}$ over $\mathbb{C}$ we denote by $\mathbf{W}$. The direct calculation gives the following commutations relations

$$
\left[k_{n}, k_{m}\right]=(n-m) k_{n+m}, \quad\left[\bar{k}_{n}, \bar{k}_{m}\right]=(n-m) \bar{k}_{n+m}, \quad\left[k_{n}, \bar{k}_{m}\right]=0 .
$$

The last relation follows from the formulas

$$
\frac{d}{d \bar{z}} z^{n}=0, \quad \frac{d}{d z} \bar{z}^{n}=0 .
$$

The algebra $\mathbf{W}$ is isomorphic two copies of the Witt algebra $W$.

Notice that $\left\{k_{0}, k_{ \pm 1}\right\}$ form a subalgebra of $\mathbf{W}$ and its projection $\left\{l_{0}, l_{ \pm 1}\right\}$ isomorphic to $\operatorname{sl}(2, C)$. The transformations of the variables $z$ and $\bar{z}$ generated by the infinitesimal operator form an infinite dimensional Lie pseudo-group $\mathbf{G}=\Gamma \otimes \bar{\Gamma}$ where $\Gamma$ and $\bar{\Gamma}$ are the infinitesimal holomorphic transformations (4.9). The conformal group of the transformations $z \mapsto z^{*}$ consists in the set Mb of Möbius transformations $\varphi$ [17]:

$$
\left(\begin{array}{ll}
a & b \\
c & d
\end{array}\right) \in \operatorname{SL}(2, C) /\{ \pm 1\} \quad \text { such that } \varphi(z)=\frac{a z+b}{c z+d}, \quad c z+d \neq 0 .
$$

For the transformations $\bar{z} \mapsto \bar{z}^{*}$ the corresponding conformal group we denote by $\overline{\mathrm{Mb}}$. The set $\mathrm{Mb}$ $(\overline{\mathrm{Mb}})$ of all global conformal transformations forms a group with respect to composition. This group operation coincides with the matrix multiplication in $\operatorname{SL}(2, C)$. $\mathrm{Mb}$ is isomorphic to the group Aut $(\mathrm{P})$ of all biholomorphic maps of the Riemann sphere $P$. It is used the compactification $\mathbb{C} \mapsto P$ to do the transition from the group $\mathrm{Mb}$ to $\operatorname{Aut}(\mathrm{P})$.

Consider the infinitesimal operator (4.10) for the signature $(+-)$ of the metric. It leads to the following relationships for the coordinates of the operator $X_{1}$

$$
\Phi_{\xi}=\Psi_{\eta}, \quad \Phi_{\eta}=\Psi_{\xi}
$$

It gives the transformation

$$
\xi^{*}=u(\xi, \eta), \quad \eta^{*}=v(\xi, \eta),
$$

with $u_{\xi}=v_{\eta}, u_{\eta}=v_{\xi}$ and generates the Lie group of orientation-preserving conformal diffeomorphisms of the Minkowski plane $\mathbb{R}_{1,1}^{2}$ (see, e.g. [17]). The group of all conformal diffeormorphisms of $\mathbb{R}_{1,1}^{2}$, endowed with the topology of uniform convergence on compact subsets, consists of four components. Each components is homeomorphic to $\operatorname{Diff}_{+}(\mathbb{R}) \times \operatorname{Diff}_{+}(\mathbb{R})$. Here Diff $+(\mathbb{R})$ denotes the group of orientation-preserving diffeomorphisms $\mathbf{F}: \mathbb{R} \mapsto \mathbb{R}$ with the topology of uniform convergence of $\mathbf{F}$ and all its derivatives on compact subsets $K \subset \mathbb{R}$. We replace $\mathbb{R}_{1,1}^{2}$ with $\mathbb{S}^{1,1}=S^{1} \times S^{1}$ 
in the sense of the conformal compactification of the Minkowski plane where $S^{1}$ is the unit circle. The conformal group $\operatorname{Conf}\left(\mathbb{R}_{1,1}^{2}\right)$ is defined as the connected components containing the identity in the group of all conformal diffeomorphisms $\mathbb{S}^{1,1} \mapsto \mathbb{S}^{1,1}$. Moreover $\operatorname{Conf}\left(\mathbb{R}_{1,1}^{2}\right)$ is isomorphic to $\operatorname{Diff}_{+}\left(S^{1}\right) \times \operatorname{Diff}_{+}\left(S^{1}\right)$. Diff $+\left(S^{1}\right)$ turns out to be a Lie group with models in the Fréchet space of smooth real-valued functions $\mathbf{F}: S^{1} \mapsto \mathbb{R}$ endowed with the uniform convergence on $S^{1}$ of $\mathbf{F}$ and all its derivatives. The corresponding Lie algebra $\operatorname{Lie}\left(\operatorname{Diff}_{+}\left(S^{1}\right)\right)$ is the Lie algebra of smooth vector space fields $\operatorname{Vect}\left(S^{1}\right)$ (see, e.g. [17]). A finite dimensional counterpart of $\operatorname{Conf}\left(\mathbb{S}^{1,1}\right)$ is the group $\mathrm{SO}(2,2) /\{ \pm 1\} \subset \operatorname{Conf}\left(\mathbb{S}^{1,1}\right)$. This group is generated by the translations and the Lorentz transformations, which form a three-dimensional subgroup, and moreover by the dilations and the special transformations. The space $C^{\infty}\left(S^{1}\right)$ can be described as the vector vector space $C_{2 \pi}^{\infty}(\mathbb{R})$ of $2 \pi$ periodic functions. A vector field $Y \in \operatorname{Vect}\left(S^{1}\right)$ has the form $Y=y(\theta) \partial / \partial \theta$, where $y(\theta) \in C_{2 \pi}^{\infty}(\mathbb{R})$ and where the points $z$ of $S^{1}$ are represented as $z=e^{i \theta}$. The representation of $y(\theta)$ by a convergent Fourier series

$$
y(\theta)=a_{0}+\sum_{n=1}^{\infty}\left(a_{n} \cos (n \theta)+b_{n} \sin (n \theta)\right)
$$

leads to basis system for $\operatorname{Vect}\left(S^{1}\right)$ :

$$
\frac{d}{d \theta}, \quad \cos (n \theta) \frac{d}{d \theta}, \quad \sin (n \theta) \frac{d}{d \theta}
$$

Consider the complexification of $\operatorname{Vect}\left(S^{1}\right)$

$$
\operatorname{Vect}^{\mathbb{C}}\left(S^{1}\right)=\operatorname{Vect}\left(S^{1}\right) \otimes \mathbb{C}
$$

and polynomial vector fields on $S^{1}$. Define

$$
\hat{l}_{n}=-z^{n+1} \frac{d}{d z}=i z^{n} \frac{d}{d \theta}=i e^{i n \theta} \frac{d}{d \theta} \in \operatorname{Vect}^{\mathbb{C}}\left(S^{1}\right)
$$

The liner hull of the $\hat{l}_{n}$ over $\mathbb{C}$ is again the Witt algebra. The Witt algebra $W$ is a part of the complexified Lie algebra $\operatorname{Vect}^{\mathbb{C}}\left(S^{1}\right) \times \operatorname{Vect}^{\mathbb{C}}\left(S^{1}\right)$. Similar as for the signature $(++)$ we can define

$$
\hat{k}_{n}=\hat{l}_{n}-(n+1) z^{n} \Lambda^{2} \frac{d}{d \Lambda^{2}}
$$

with the same rule of transformation of the factor $\Lambda^{2}$. The commutation relations for $\hat{k}_{n}$ and $\hat{k}_{m}$ are the same as before for $k_{n}$ and $k_{m}$.

\subsection{Invariant differentiations}

Now we define the operators of invariant differentiations. Below we use the symbols ++ and +which corresponds to the consideration of $\mathbf{G}_{++}$and $\mathbf{G}_{+-}$. In the variables $\xi$ and $\eta$, the operators of 
invariant differentiations are determined by the formula [14]

$$
S_{++}^{i}=\vec{\lambda}^{i} \cdot\left(D_{\xi}+D_{\eta}\right), \quad S_{+-}^{i}=\vec{\mu}^{i} \cdot\left(D_{\xi}+D_{\eta}\right), \quad D=D_{\xi}+D_{\eta}, \quad i=1,2,
$$

where the vectors $\vec{\lambda}^{i}$ and $\vec{\mu}^{i}$ are calculated from the equations

$$
J^{++}\left(\vec{\lambda}^{i}\right)=\mathbf{c}^{\mathbf{i}}, \quad J^{+-}\left(\vec{\mu}^{i}\right)=\mathbf{c}^{\mathbf{i}}, \quad \mathbf{c}^{i} \in \mathbb{R}^{3},
$$

$\mathbf{c}^{\mathbf{i}}$ are chosen to be $\mathbf{c}^{1}=(0,1,0), \mathbf{c}^{2}=(0,1,1) . J^{++}$and $J^{+-}$are found as solutions of the equations

$$
\begin{aligned}
& {\left[X_{1}^{++}+\left(\vec{\lambda}^{i} \cdot D\right) \vec{\chi} \cdot \partial_{\lambda}\right] J^{++}=0,} \\
& {\left[X_{1}^{+-}+\left(\vec{\mu}^{i} \cdot D\right) \vec{\chi} \cdot \partial_{\mu}\right] J^{+-}=0,}
\end{aligned}
$$

where $\partial_{\lambda}=\lambda_{1} \partial / \partial \lambda_{1}+\lambda_{2} \partial / \partial \lambda_{2}$ and correspondingly $\partial_{\mu}=\mu_{1} \partial / \partial \mu_{1}+\mu_{2} \partial / \partial \mu_{2}$. Here $\vec{\chi}=$ $(\Phi, \Psi, 0), X_{1}^{++}$and $X_{1}^{+-}$denote the first prolongations of the operator $X_{1}$ for the signatures $(++)$ and $(+-)$ correspondingly. These operators read

$$
\begin{aligned}
& X_{1}^{++}=\Phi \frac{\partial}{\partial \xi}+\Psi \frac{\partial}{\partial \eta}-\Phi_{\xi}\left(2 \Lambda^{2} \frac{\partial}{\partial \Lambda^{2}}+\tau_{\xi} \frac{\partial}{\partial \tau_{\xi}}+\tau_{\eta} \frac{\partial}{\partial \tau_{\eta}}+3 \Lambda_{\xi}^{2} \frac{\partial}{\partial \Lambda_{\xi}^{2}}+3 \Lambda_{\eta}^{2} \frac{\partial}{\partial \Lambda_{\eta}^{2}}\right) \\
& -\Psi_{\xi}\left(\tau_{\eta} \frac{\partial}{\partial \tau_{\xi}}-\tau_{\xi} \frac{\partial}{\partial \tau_{\eta}}+\Lambda_{\eta}^{2} \frac{\partial}{\partial \Lambda_{\xi}^{2}}-\Lambda_{\xi}^{2} \frac{\partial}{\partial \Lambda_{\eta}^{2}}\right)-2 \Phi_{\xi \xi} \Lambda^{2} \frac{\partial}{\partial \Lambda_{\xi}^{2}}-2 \Phi_{\xi \eta} \Lambda^{2} \frac{\partial}{\partial \Lambda_{\eta}^{2}}
\end{aligned}
$$

and

$$
\begin{aligned}
& X_{1}^{+-}=\Phi \frac{\partial}{\partial \xi}+\Psi \frac{\partial}{\partial \eta}-\Phi_{\xi}\left(2 \Lambda^{2} \frac{\partial}{\partial \Lambda^{2}}+\tau_{\xi} \frac{\partial}{\partial \tau_{\xi}}+\tau_{\eta} \frac{\partial}{\partial \tau_{\eta}}+3 \Lambda_{\xi}^{2} \frac{\partial}{\partial \Lambda_{\xi}^{2}}+3 \Lambda_{\eta}^{2} \frac{\partial}{\partial \Lambda_{\eta}^{2}}\right) \\
& -\Psi_{\xi}\left(\tau_{\eta} \frac{\partial}{\partial \tau_{\xi}}+\tau_{\xi} \frac{\partial}{\partial \tau_{\eta}}+\Lambda_{\eta}^{2} \frac{\partial}{\partial \Lambda_{\xi}^{2}}-\Lambda_{\xi}^{2} \frac{\partial}{\partial \Lambda_{\eta}^{2}}\right)-2 \Phi_{\xi \xi} \Lambda^{2} \frac{\partial}{\partial \Lambda_{\xi}^{2}}-2 \Phi_{\xi \eta} \Lambda^{2} \frac{\partial}{\partial \Lambda_{\eta}^{2}}
\end{aligned}
$$

wherein we used the Cauchy-Riemann conditions $\Phi_{\xi}=\Psi_{\eta}, \Phi_{\eta}=-\Psi_{\xi}$ for the operator $X_{1}^{++}$and the $h$-holomorphic conditions $\Phi_{\xi}=\Psi_{\eta}, \Phi_{\eta}=\Psi_{\xi}$ in the case of the operator $X_{1}^{+-}$. Equation (4.13) has the following two functionally independent solutions

$$
J_{1}^{++}=\Lambda^{2}\left(\lambda_{1}^{2}+\lambda_{2}^{2}\right), \quad J_{2}^{++}=\lambda_{1} \tau_{\eta}-\lambda_{2} \tau_{\xi},
$$

the vectors $\vec{\lambda}^{i}$ are defined by

$$
\vec{\lambda}^{1}=\left(\frac{\tau_{\xi}}{\Lambda^{2}}, \frac{\tau_{\eta}}{\Lambda^{2}}, 0\right), \quad \vec{\lambda}^{2}=\left(\frac{\tau_{\eta}}{\Lambda^{2}},-\frac{\tau_{\xi}}{\Lambda^{2}}, 0\right) .
$$

What about equation (4.14), we have the following two functionally independent solutions

$$
J_{1}^{+-}=\Lambda^{2}\left(\mu_{1}^{2}-\mu_{2}^{2}\right), \quad J_{2}^{+-}=\mu_{1} \tau_{\eta}+\mu_{2} \tau_{\xi},
$$

and the vectors $\vec{\mu}^{i}$ equal

$$
\vec{\mu}^{1}=\left(\frac{\tau_{\xi}}{\Lambda^{2}},-\frac{\tau_{\eta}}{\Lambda^{2}}, 0\right), \quad \vec{\mu}^{2}=\left(-\frac{\tau_{\eta}}{\Lambda^{2}}, \frac{\tau_{\xi}}{\Lambda^{2}}, 0\right) .
$$


We give the following comments to these relationships obtained. Let us consider nontrivial solutions of the equation

$$
J_{1}^{+-}\left(\vec{\mu}^{1}\right) \equiv \Lambda^{2}\left(\mu_{1}^{2}-\mu_{2}^{2}\right)=1, \quad\left(\text { the second component of } \mathbf{c}^{1}\right),
$$

then inserting the vector $\vec{\mu}^{1}$ into (4.15), we get

$$
\tau_{\xi}^{2}-\tau_{\eta}^{2}=\Lambda^{2}
$$

therefore equation (4.15) is satisfied identically. The same arguments hold for the function $J_{2}^{+-}\left(\vec{\mu}^{2}\right)$ and the vector $\vec{\mu}^{2}$.

As a result, we obtain the operators of invariant differentiations

$$
\begin{gathered}
S_{1}^{++}=\frac{1}{\Lambda^{2}}\left(\tau_{\xi} D_{\xi}+\tau_{\eta} D_{\eta}\right), \quad S_{2}^{++}=\frac{1}{\Lambda^{2}}\left(\tau_{\eta} D_{\xi}-\tau_{\xi} D_{\eta}\right), \\
S_{1}^{+-}=\frac{1}{\Lambda^{2}}\left(\tau_{\xi} D_{\xi}-\tau_{\eta} D_{\eta}\right), \quad S_{2}^{+-}=\frac{1}{\Lambda^{2}}\left(-\tau_{\eta} D_{\xi}+\tau_{\xi} D_{\eta}\right),
\end{gathered}
$$

where $S_{2}^{++}=-S_{2}^{+-}$.

The scalar invariants of the first-order of $\mathbf{G}_{++}$and $\mathbf{G}_{+-}$are defined by

$$
X_{1}^{++} I^{++}=0, \quad X_{1}^{+-} I^{+-}=0 .
$$

Splitting these equations, we can easily find the following functional-independent solutions

$$
\begin{array}{ll}
I_{1}^{++}=\tau, & I_{2}^{++}=\frac{1}{\Lambda^{2}}\left(\tau_{\xi}^{2}+\tau_{\eta}^{2}\right) \\
I_{1}^{+-}=\tau, & I_{2}^{+-}=\frac{1}{\Lambda^{2}}\left(\tau_{\xi}^{2}-\tau_{\eta}^{2}\right)
\end{array}
$$

Therefore the universal first-order differential invariants $J_{1}^{++}$and $J_{1}^{+-}$of the Lie pseudo-group $\mathbf{G}_{++}$ and Lie group $\mathbf{G}_{+-}$are of the form

$$
J_{1}^{++}=\left(I_{1}^{++}, S_{1}^{++} I_{1}^{++}\right), \quad J_{1}^{+-}=\left(I_{1}^{+-}, S_{1}^{+-} I_{1}^{+-}\right), \quad I_{1}^{++}=I_{1}^{+-} .
$$

The actions of $S_{2}^{++}$and $S_{2}^{+-}$on $I_{1}^{++}$and $I_{1}^{+-}$correspondingly lead to preserving the length of the tangent vector $(\xi, \eta)$ to the geodesic curves of length equals $\tau$. It means that the geodesic curves under the action of $\mathbf{G}_{++}$and $\mathbf{G}_{+-}$transformed into the geodesic curves.

According to Tresse theorem [14], using the operators $S_{i}^{++}$and $S_{j}^{++}, i, j=1,2$ and the scalar invariant $I_{1}^{++}=I_{1}^{+-}=\tau$, we can construct finite basis of differential invariants of $\mathbf{G}_{++}$and $\mathbf{G}_{+-}$by functional operations and the invariant differentiation. In particular, the Gaussian curvature $K$ reads

$$
K_{++}=-\frac{1}{2} \frac{\Delta \ln \Lambda^{2}}{\Lambda^{2}} \quad \text { for } M_{I_{1}}^{t} \quad \text { and } K_{+-}=\frac{1}{2} \frac{\Delta \ln \Lambda^{2}}{\Lambda^{2}} \quad \text { for } M_{I_{i}}^{t}, i=2,3
$$

and $K_{++}, K_{+-}$are the differential invariants of the second order of $\mathbf{G}_{++}$and $\mathbf{G}_{+-}$correspondingly. This fact is checked by direct calculations which show that

$$
X_{2}^{++} K_{++}=0, \quad X_{2}^{+-} K_{+-}=0
$$

where $X_{2}^{++}$and $X_{2}^{+-}$denotes the second prolongation of the operator $X$ for the signature $(++)$ and $(+-)$ correspondingly. These two differential invariants $K_{++}$and $K_{+-}$can be expressed in the form

$$
K_{++}=V\left(S_{i}^{++} I_{1}^{++}\right) \quad K_{+-}=-V\left(S_{i}^{+-} I_{1}^{+-}\right), i=1,2
$$


where $V\left(S_{i}^{++} I_{1}^{++}\right)$and $V\left(S_{i}^{+-} I_{1}^{+-}\right)$are some differential-algebraic expressions. Due to the formula (2.11) which connects $K_{++}$and the Taylor microscale $\lambda_{g}$ and the result above, we can claim that the Taylor microscale is a differential invariant of $\mathbf{G}_{++}$.

\subsection{Local shape structure of $M_{I_{i}}^{t}$}

Consider the manifold $M_{I_{1}}^{t}$. We showed that in every chart of $M_{I_{1}}^{t}$ acts the Lie pseudo-group $\mathbf{G}_{++}$ that preserves the form of the metric and generates infinitesimal deformations of the surface $M_{I_{1}}^{t}$. This deformation is evoked by the infinitesimal transformations

$$
\xi^{*}=\xi+\delta \xi, \quad \eta^{*}=\eta+\delta \eta,
$$

or in the complex variables

$$
z^{*}=z-\sum_{n=-\infty}^{\infty} \varepsilon_{n} z^{n}, \quad \bar{z}^{*}=z-\sum_{n=-\infty}^{\infty} \varepsilon_{n} \bar{z}^{n}
$$

Therefore we have infinite number of transformations of the form

$$
z_{n}^{*}=z-\varepsilon_{n} z^{n}, \quad \bar{z}_{n}^{*}=\bar{z}-\varepsilon_{n} \bar{z}^{n} .
$$

We establish that these transformations generates sliding $M_{I_{1}}^{t}$ along itself. In other words there is no deformations of $M_{I_{1}}^{t}$ in the direction of normal vector to the surface $M_{I_{1}}^{t}$ at the corresponding points of this deformation. In order to prove this assertion assume the contrary i.e. we can think that

$$
\vec{U}(\xi, \eta)=h \cdot \vec{n}+g \cdot \vec{k},
$$

where the second term responses for sliding $M_{I_{1}}^{t}$ along itself. Hence we can write $\vec{r}^{*}=\overrightarrow{\tilde{r}}+h \cdot \vec{n}$ wherein $\overrightarrow{\vec{r}}=\vec{r}$ after sliding. For $\vec{r}^{*}=\vec{r}^{*}\left(\vec{\xi}^{*}, \vec{\eta}^{*}\right)$ the metric reads

$$
\begin{aligned}
d s^{* 2}= & \left(\Lambda^{* 2}+2 h L+\left(2 H L-K \Lambda_{1}^{* 2}\right) h^{2}+h_{\xi^{*}}^{2}\right) d \xi^{* 2}+2\left(2 h M+2 H M h^{2}+\right. \\
& \left.h_{\xi^{*} \eta^{*}}\right) d \xi^{* 2} d \eta^{* 2}+\left(\Lambda^{* 2}+2 h N+\left(2 H N-K \Lambda^{* 2}\right) h^{2}+h_{\eta^{*}}^{2}\right) d \eta^{* 2} .
\end{aligned}
$$

Here $H, K$ are the average and Gaussian curvature, $L, M$ and $N$ are the coefficients of the second quadratic form. The conformal transformations conserve the form of metrics, it means that we have $h \equiv 0$. This factum also follows from the well-known result about the transformation of metrics to the canonical (conformal) form by a quasi-conformal mapping $\omega=\omega(z), z=\xi+i \eta$ which satisfies the Beltrami equation

$$
\frac{\partial \omega}{\partial \bar{z}}=p(z) \frac{\partial \omega}{\partial z}
$$

where

$$
\begin{aligned}
& p(z)=\frac{(L-N+2 i M) h+H(L-N+2 i M) h^{2}+2(\partial h / \partial \bar{z})^{2}}{\left(1+2 H h+\left(2 H^{2}-K\right) h^{2}+2|\partial h / \partial z|^{2}\right) \Lambda_{1}^{-2}+\sqrt{\Delta} \Lambda_{1}^{2}} \\
\Delta= & 1+4 H h+\left(4 H^{2}+2 K\right) h^{2}+4|\partial h / \partial z|^{2} \Lambda_{1}^{-2} \\
+ & 4 K H h^{3}+K^{2} h^{4}+2\left(L h_{\eta}-2 M h_{\xi} h_{\eta}\right. \\
+ & \left.N h_{\xi}^{2}\right) \Lambda_{1}^{-4}+\left(\left(2 H L-K \Lambda_{1}^{2}-4 H M h_{\eta} h_{\xi}+\left(2 H N-K \Lambda_{1}^{2}\right) h_{\xi}^{2}\right) h^{2} \Lambda_{1}^{-4}\right.
\end{aligned}
$$


and

$$
\Lambda^{* 2}\left(\xi^{*}, \eta^{*}\right)=\Lambda^{2}(\xi, \eta) \frac{1+2 H h+\left(2 H^{2}-K\right) h^{2}+2|\partial h / \partial z|^{2} \Lambda_{1}^{-2}+\sqrt{\Delta}}{2\left(\partial z^{*} / \partial z\right) \cdot\left(\partial \bar{z}^{*} / \partial \bar{z}\right)}
$$

The conformal property of the transformation $\xi \mapsto \xi^{*}, \eta \mapsto \eta^{*}$ (or $z \mapsto z^{*}, \bar{z} \mapsto \bar{z}^{*}$ ) leads to vanishing $p$ and therefore $h=0$. Here $\Lambda^{2}(\xi, \eta)$ is transformed to $\Lambda^{* 2}\left(\xi^{*}, \eta^{*}\right)$ according to the formula

$$
\Lambda^{* 2}\left(\xi^{*}, \eta^{*}\right)=\frac{\Lambda^{2}(\xi, \eta)}{\left(\partial z^{*} /(\partial z) \cdot\left(\partial \bar{z}^{*} / \partial \bar{z}\right)\right.}
$$

which coincides with (4.8) and completes the proof.

This assertion we use to classify the shape of $M_{I_{1}}^{t}$ at least locally. The tangent components of the vector of displacement $\vec{U}(\xi, \eta)$ we denote by $u_{\alpha}, \alpha=1,2$ and then $\vec{U}=u_{\alpha} \vec{\tau}^{\alpha}$ where $\vec{\tau}^{1}=(1,0)$, $\vec{\tau}^{2}=(0,1)$. The kinematic system of equations of the field of displacements in the case of sliding $M_{I_{1}}^{t}$ along itself is of the form (see for details [18])

$$
\nabla_{\alpha} u_{\beta}+\nabla_{\beta} u_{\alpha}=0, \quad \alpha, \beta=1,2,
$$

where $\nabla_{\alpha} u_{\beta}=\partial u_{\beta} / \partial x^{\alpha}-\Gamma_{\alpha \beta}^{\lambda} u_{\lambda}$. We introduce into the consideration the complex function of displacement $w=u_{1}+i u_{2}$ and define for the positive Gaussian curvature $K_{++}>0$ the function

$$
W=\frac{w(z)}{\sqrt{a K^{1 / 2}}}, \quad a=a_{11} a_{22}-a_{12}^{2}>0, \quad a_{\alpha \beta}=\vec{r}_{\alpha} \vec{r}_{\beta}, \quad \vec{r}_{1}=\frac{\partial \vec{r}}{\partial \xi}, \quad \vec{r}_{2}=\frac{\partial \vec{r}}{\partial \eta}
$$

which satisfies the equation [18]

$$
\partial_{\bar{z}} W+B \bar{W}=0 .
$$

Here

$$
B=\frac{1}{4}\left(\Gamma_{22}^{1}-\Gamma_{11}^{1}+2 \Gamma_{12}^{2}\right)-\frac{i}{4}\left(\Gamma_{11}^{2}-\Gamma_{22}^{2}+2 \Gamma_{12}^{1}\right) .
$$

The derivation of equation (4.20) from the kinematic system of equations (4.19) is given in [18]. The direct calculations of the Cristoffel symbols in (4.21) show that

$$
\frac{1}{4}\left(\Gamma_{22}^{1}-\Gamma_{11}^{1}+2 \Gamma_{12}^{2}\right)=0, \quad \frac{i}{4}\left(\Gamma_{11}^{2}-\Gamma_{22}^{2}+2 \Gamma_{12}^{1}\right)=0 .
$$

Therefore the function $W$ is a holomorphic function and the complex function of displacement $w=w(z)$ reads

$$
w(z)=\sqrt{a K_{++}^{1 / 2}} \Theta(z),
$$

where $\Theta(z)$ is a holomorphic function. In the case when $W_{I_{1}}^{t}$ is a part of the spherical surface then $w(z)=(1+z \bar{z})^{-2} \Theta(z)$. In general the following classification theorem holds

Theorem 4.1 ([18]). The condition $B \equiv 0$ is satisfied for the second-order algebraic surfaces of a positive Gaussian curvature and only for such surfaces.

As a corollary of this theorem, we can claim that in each chart where $M_{I_{1}}^{t}$ has a positive Gaussian curvature the surface $M_{I_{1}}^{t}$ takes locally the form of a part of ellipsoid (in particular sphere), bicameral hyperboloid or paraboloid. Notice that for $K_{++}<0$ there is no similar result to classify surfaces of 
negative Gaussian curvature. It is well-known that each second-order algebraic surface of revolution with positive Gauss curvature $K_{++}>0$ can be transformed by an infinitesimal pressure along any parallel into the surface of variable curvature. If this infinitesimal pressure is not scaling then we get a surface which different from the second-order algebraic surfaces.

Consider now the manifolds $M_{I_{j}}^{t}, j=2,3$ with the metric of (+-) signature. For convenience, we introduce new coordinates (which are standard in CFT)

$$
\xi^{+}=\xi+\eta, \quad \xi^{-}=\xi-\eta .
$$

This change of coordinates is simply a rotation on the angle $\pi / 2$. Let us perform a Wick rotation $\eta \mapsto i \eta, i^{2}=-1$ that results in

$$
\xi^{+} \mapsto z=\xi+i \eta, \quad \xi^{-} \mapsto \bar{z}=\xi-i \eta,
$$

with the metric $d s^{2}=\Lambda^{2}(z \bar{z}) d z d \bar{z}$. Denote by $i M_{I_{j}}^{t}, i^{2}=-1, j=2,3$ a transformation of $M_{I_{j}}^{t}$ under the Wick rotation, we can look on $i M_{I_{j}}^{t}$ as the complex realization of $M_{I_{j}}^{t}$ in the new chart $(z, \bar{z})$. The classification of $M_{I_{j}}^{t}$ can be formulated in the terms of $i M_{I_{j}}^{t}$ applying the reasoning above.

\section{Asymptotic Expansion of the Transversal Correlation Function}

Consider in $K^{3}$ an infinite cylindrical domain. Fix the cross-section $\{0\} \times D^{2}$ of this domain where $D^{2}$ is a two-dimensional disk. Then the quadratic form $d l^{2}(t)$ induces on the above-mentioned cross-section the metric

$$
d l_{D^{2}}^{2}(t)=\overline{u^{\prime 2}(t)} g(|\vec{r}|, t)\left(d r^{2^{2}}+d r^{3^{2}}\right),
$$

where $|\vec{r}|=\sqrt{r^{2^{2}}+r^{3^{3}}}$. Consider the set $\Omega\left(D^{2}, v, w\right)$ of piecewise smooth curves $\gamma: J \rightarrow D^{2}$ with fixed endpoints $\gamma(0)=v$ and $\gamma(1)=w$ and the functional of action of the trajectory $\gamma$

$$
E_{\gamma}^{t}=\int_{\gamma} u^{2}\left(r_{\tau}^{2}+r_{\tau}^{3^{2}}\right) d \tau
$$

We can rewrite it in the following form

$$
E_{\gamma}^{t}=\int_{\gamma}\left(u^{2}\right)^{-1}\left(\tau_{r^{2}}^{2}+\tau_{r^{3}}^{2}\right) d \tau, \quad \tau_{r^{2}}^{2}+\tau_{r^{3}}^{2}=u^{2},
$$

where $u^{2}=\overline{u^{\prime 2}(t)} g(|\vec{r}|, t)$. It takes the form in the complex variables frame

$$
E_{\gamma}^{t}=4 \int_{\gamma}\left(u^{2}\right)^{-1}(z \bar{z}, t) \partial_{z} \tau(z, \bar{z}) \cdot \partial_{\bar{z}} \tau(z, \bar{z}) d \tau
$$

The classical energy-momentum tensor is defined by

$$
T_{i k}=g_{k l} f_{x^{i}}^{\alpha} \frac{\partial L}{\partial f_{x^{l}}^{\alpha}}-g_{i k} L
$$

where $L=L\left(f^{j}, f_{x^{k}}^{i}\right)$ is a Lagrangian. For the functional (5.2) with the scalar field $\tau$ this tensor takes the form

$$
T_{11}=2 \tau_{r^{2}}^{2}-u^{2} \cdot 1, \quad T_{22}=2 \tau_{r^{3}}^{2}-u^{2} \cdot 1, \quad T_{12}=T_{21}=2 \tau_{r^{2}} \tau_{r^{3}}
$$


$T_{i k}$ is a traceless tensor due to the equality $T_{11}+T_{22}=2\left(\tau_{r^{2}}^{2}+\tau_{r^{3}}^{2}-u^{2}\right)=0$. Denote by $\vec{\omega}=\left(r^{2}, r^{3}\right)$ and $\overrightarrow{\delta \omega}=\left(\delta r^{2}, \delta r^{3}\right)$. Then the current is defined by $j_{\mu}=T_{\mu \nu} \delta \omega^{v}$ and has an automatically vanishing divergence $\partial \cdot j=(1 / 2) T_{\mu}^{\mu}(\partial \cdot \delta \omega)$ due to the traceless condition on $T_{\mu \nu}$. Since $T_{i k}$ is traceless, we have $T_{z \bar{z}}=T_{\bar{z} z}=0$ in the complex variables. These imply $\partial_{\bar{z}} T_{z z}=\partial_{z} T_{\bar{z} \bar{z}}=0$ and there are only two non-vanishing components of the energy-momentum tensor $T(z)=T_{z z}(z)=1 / 4\left(T_{11}-2 i T_{12}-T_{22}\right)$ and $\bar{T}(\bar{z})=T_{\bar{z} \bar{z}}(\bar{z})=1 / 4\left(T_{11}+2 i T_{12}-T_{22}\right)$. Moreover $T_{z z}=\left(\partial_{z} \tau\right)^{2}$ and $T_{\bar{z} \bar{z}}=\left(\partial_{\bar{z}} \tau\right)^{2}$. In the line of CFT, we can introduce into the consideration the following constructions: since $T(z)$ and $\bar{T}(\bar{z})$ are holomorphic functions then due to Laurent expansions

$$
T(z)=\sum_{n} L_{n} z^{-n-2}, \quad \bar{T}(\bar{z})=\sum_{n} \bar{L}_{n} \bar{z}^{-n-2},
$$

where the exponent $-n-2$ is chosen so that for the scale transformation $z \mapsto \lambda^{-1} z$ under which $T(z) \mapsto \lambda^{2} T\left(\lambda^{-1} z\right)$ we have $L_{-n} \mapsto \lambda^{n} L_{-n}$, and $\bar{L}_{-n} \mapsto \bar{\lambda}^{n} \bar{L}_{-n}$. Therefore, the expressions (5.6) is finally inverted by the relations

$$
L_{n}=\frac{1}{2 \pi i} \oint T(z) z^{n+1} d z, \quad \bar{L}_{n}=\frac{1}{2 \pi i} \oint \bar{T}(\bar{z}) \bar{z}^{n+1} d \bar{z} .
$$

Recall that $L_{n}$ are defined on $\mathbb{C} \backslash\{0\}$ and consider

$$
T_{\varepsilon}=\oint \varepsilon(z) T(z) d z, \quad \bar{T}_{\bar{\varepsilon}}=\oint \bar{\varepsilon}(\bar{z}) \bar{T}(\bar{z}) d z
$$

where

$$
\varepsilon(z)=-\sum_{n=-\infty}^{\infty} \varepsilon_{n} z^{n+1}, \quad \bar{\varepsilon}(\bar{z})=-\sum_{n=-\infty}^{\infty} \bar{\varepsilon}_{n} \bar{z}^{n+1}
$$

and $\varepsilon_{n}\left(\bar{\varepsilon}_{n}\right)$ are infinitesimal small numbers. Their variations read [1]

$$
\begin{aligned}
& \delta_{\varepsilon} T(z)=\varepsilon(z) T^{\prime}+2 \varepsilon^{\prime}(z) T(z)+(1 / 12) c \varepsilon^{\prime \prime \prime}(z), \\
& \delta_{\bar{\varepsilon}} \bar{T}(z)=\bar{\varepsilon}(\bar{z}) \bar{T}^{\prime}+2 \bar{\varepsilon}^{\prime}(\bar{z}) \bar{T}(\bar{z})+(1 / 12) \bar{c} \bar{\varepsilon}^{\prime \prime \prime}(\bar{z}),
\end{aligned}
$$

It leads to the commutation relations [1]

$$
\begin{aligned}
& {\left[T_{\mathcal{\varepsilon}}, T(z)\right]=\varepsilon(z) T^{\prime}(z)+2 \varepsilon(z) T(z)+\frac{c}{12} \varepsilon^{\prime \prime \prime}(z),} \\
& {\left[\bar{T}_{\bar{\varepsilon}}, \bar{T}(\bar{z})\right]=\bar{\varepsilon}(\bar{z}) \bar{T}^{\prime}(\bar{z})+2 \bar{\varepsilon}(\bar{z}) \bar{T}(\bar{z})+\frac{c}{12} \bar{\varepsilon}^{\prime \prime \prime}(\bar{z}) .}
\end{aligned}
$$

Then from (5.7),(5.8) follow that $L_{n}$ and $\bar{L}_{n}$ satisfy the commutation relations [1]

$$
\begin{gathered}
{\left[L_{n}, L_{m}\right]=(n-m) L_{n+m}+\frac{c}{12} \delta_{n+m} n\left(n^{2}-1\right),} \\
{\left[\bar{L}_{n}, \bar{L}_{m}\right]=(n-m) \bar{L}_{n+m}+\frac{\bar{c}}{12} \delta_{n+m} n\left(n^{2}-1\right), \quad\left[L_{n}, \bar{L}_{m}\right]=0 .}
\end{gathered}
$$

This algebra is the Lie algebra of the central extension of the group of diffeomorphisms of the circle whose basic elements $\left\{L_{n}\right\}, n \in Z$. Here $\delta_{k}=1$ for $k=0$ and $\delta_{k}=0$ for $k \neq 0$. The quantity $c$ is known as the central charge. 
Theorem 5.1 ([1]). Let $T(z)=\sum_{n} L_{n} z^{-n-2}$ and $T(w)=\sum_{n} L_{n} w^{-n-2}$ be the fields with $\left\{L_{n}\right\}$ satisfying the commutation relationship (5.9). Then for large $z$ and $w$ it holds

$$
T(z) T(w) \sim \frac{c}{2(z-w)^{4}}+\frac{2 T(w)}{(z-w)^{2}}+\frac{\partial_{w} T(w)}{z-w} .
$$

The proof of this Theorem is based on the direct calculations. The first term of the right-hand side of (5.9) equals

$$
(n-m) L_{n+m}=(n-m) \oint \frac{d w}{2 \pi i}\left(w^{n+m+1} T(w)\right) .
$$

The left-hand side of (5.9) reads

$$
\left[L_{n}, L_{m}\right]=\left(\oint_{|z|>|w|} \frac{d z}{2 \pi i} \oint \frac{d w}{2 \pi i}-\oint \frac{d w}{2 \pi i} \oint_{|z|<|w|} \frac{d z}{2 \pi i}\right) z^{n+1} T(Z) w^{n+1} T(w) .
$$

This integral can be rewritten as

$$
\oint \frac{d w}{2 \pi i}\left(2(n+1) w^{n} w^{m+1} T(w)+w^{n+1} w^{m+1} \partial_{w} T(w)\right) .
$$

Indeed, integrating the last term of this integral by parts and combining with the first term we get the right-hand part of (5.12). The second term of (5.9) that is $\frac{c}{12} \delta_{n+m} n\left(n^{2}-1\right)$ can be presented as the following integral

$$
\oint \frac{d w}{2 \pi i}\left(\frac{c}{12} n\left(n^{2}-1\right) w^{n-2} w^{m}\right) .
$$

Further transformations are based on using the well-known formula from the Complex Analysis

$$
\partial_{\zeta}^{n} f(\zeta)=n ! \oint \frac{d w}{2 \pi i} \frac{f(\zeta)}{(\zeta-z)^{n}}
$$

Since $\left.\partial_{z}^{3} z^{n+1}\right|_{z=w}=n\left(n^{2}-1\right) w^{n-2}$ then applying (5.16), we get that (5.15) equals

$$
\oint \frac{d z}{2 \pi i} \oint \frac{d w}{2 \pi i} z^{n+1} w^{m+1} \frac{c}{2(z-w)^{4}}
$$

Applying the same procedure to the first and the second terms of (5.14), we can see that this integral equals

$$
\oint \frac{d z}{2 \pi i} \oint \frac{d w}{2 \pi i} z^{n+1} w^{m+1}\left(\frac{2 T(w)}{(z-w)^{2}}+\frac{\partial T(w)}{z-w}\right) .
$$

Combining (5.17) and (5.18), we found that the integral

$$
\oint \frac{d z}{2 \pi i} \oint \frac{d w}{2 \pi i} z^{n+1} w^{m+1}\left(\frac{c}{2(z-w)^{4}}+\frac{2 T(w)}{(z-w)^{2}}+\frac{\partial T(w)}{z-w}\right)
$$

equals the right-hand side of (5.13). For the completeness, we need to add at the integral (5.19) a regular function $R(z, w)$ on the variable $z-w$ since this function are free of poles at $z=w$, hence 
$R(z, w)$ does not contribute to the integral. Therefore comparing (5.19) with (5.13), it is derived that

$$
T(z) T(w)=\frac{c}{2(z-w)^{4}}+\frac{2 T(w)}{(z-w)^{2}}+\frac{\partial_{w} T(w)}{z-w}+R(z, w) .
$$

This relationship is called the operator product expansion in CFT. The same assertion are hold for the $\bar{T}(\bar{z})$. Notice that the quantities $c$ and $\bar{c}$ coincide.

\section{Corollary 5.1 .}

$$
T(z) T(0) \sim \frac{c}{2 z^{4}}, \quad \bar{T}(\bar{z}) \bar{T}(0) \sim \frac{c}{2 \bar{z}^{4}} \quad \text { as } z, \bar{z} \rightarrow \infty .
$$

We apply (5.20) to find asymptotic expansion the transversal correlation function $B_{N N}=$ $\overline{u^{\prime 2}(t)} g(z \bar{z})$ as $|\vec{r}| \rightarrow \infty$. For this, we use the following relationship

$$
4 \partial_{z} \tau \cdot \partial_{\bar{z}} \tau=u^{2}
$$

and the equalities $T(z)=T_{z z}=\left(\partial_{z} \tau\right)^{2}$ and $\bar{T}(\bar{z})=T_{\bar{z} \bar{z}}=\left(\partial_{\bar{z}} \tau\right)^{2}$. Rewrite (5.21) in the form $\left(\partial_{z} \tau\right)^{2}$. $\left(\partial_{\bar{z}} \tau\right)^{2}=(1 / 16)\left(u^{2}\right)^{2}$ and use the formulas (5.20). Then substituting instead of $\left(u^{2}\right)^{2}$ the quantity ${\overline{u^{\prime 2}(t)}}^{2} g^{2}(|\vec{r}|, t)$ in (5.20), we get the asymptotic expansion for $B_{N N}^{2}(|\vec{r}|, t)$ in the form

$$
{\overline{u^{\prime 2}(t)}}^{2} g^{2}(|\vec{r}|, t) \sim \frac{4 c^{2}}{|\vec{r}|^{8} T(0) \bar{T}(0)}, \quad z \bar{z}=|\vec{r}|^{2} \quad \text { as }|\vec{r}| \rightarrow \infty .
$$

Since $T(0) \bar{T}(0)=(1 / 16){\overline{u^{\prime 2}(t)}}^{2}$, we finally obtain from (5.22) that

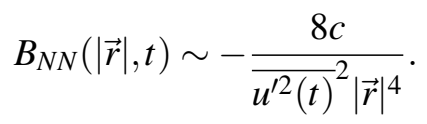

The sign "-" appears in view of the negative values of $B_{N N}(|\vec{r}|, t)$ as $|\vec{r}| \rightarrow \infty$.

We presented the asymptotic behavior of the transversal correlation function $B_{N N}$ as $|\vec{r}| \rightarrow \infty$ and this is in $K^{3}$ with $d l_{D^{2}}^{2}(t)$, see [6] for more details.

\section{References}

[1] A.A. Belavin, A.M. Polyakov, A.B. Zamolodchikov, Infinite conformal symmetry in two-dimensional quantum field theory, Nucl. Phys. B 241 (1984) 333-380.

[2] P.A. Davidson, Turbulence. An introduction for scientists and engineers (Oxford Univiersity Press, 2004).

[3] V.N. Grebenev, M. Oberlack, Geometric realization of the two-point correlation tensor for isotropic turbulence, J. Nonl. Math. Phys. 18(1) (2011) 109-120.

[4] V.N. Grebenev, M. Oberlack, A geometry of the correlation space and a nonlocal degenerate parabolic equation from isotropic turulence. ZAMM. Z. Angew. Math. Mech. 92(3) (2012) 179-195.

[5] V.N. Grebenev, M. Oberlack, A.N. Grishkov, Infinite dimensional Lie algebra associated with the twopoint velocity correlation tensor from isotropic, ZAMP, Z. Angew. Math. Phys. 64 (2013) 599-620.

[6] V.N. Grebenev, A.N. Grishkov, M. Oberlack, The extended symmetry Lie algebra and the asymptotic expansion of the transversal correlation function for isotropic turbulence, Adv. Math. Phys. 2013 article ID 46954 (2013) $11 \mathrm{pp}$.

[7] K. Hasselmann, Zur Deutung der dreifachen Geschwindigkeits korrelationen der isotropen Turbulenz, Dtsh. Hydrogr. Zs. 11 (1958) 207-211.

[8] N.R. Kamyshanskij and A.S. Solodovnikov, Semireducible analytic spaces "in the large", Russ. Math. Surv. 35(5) (1980) 1-56. 
[9] Th. von Kármán, L. Howarth, On the statistical theory of isotropic turbulence, Proc. Roy. Soc. A164 (1938) 192-215.

[10] A.M. Lavrentjev and B.V. Shabat, Problems of Hydrodynamics (Nauka, Moscow, 1973).

[11] A.S. Monin and A.M. Yaglom, Statistical Hydromechanics (Gidrometeoizdat, St.-Petersburg, 1994).

[12] A.G. Megrabov, Group spliting and Lax representation, Dokl. Math. 67 (3) (2003) 335-349.

[13] S.V. Meleshko, Homogeneous autonomic systems with three independent variables, J. Appl. Math. Mech. 58 (1994) 857-863.

[14] L.V. Ovsynnikov, Group analysis of differential equations (Nauka, Moscow, 1978).

[15] S. Oughton, K.-H. Rädler and W.H. Matthaeus, General second-rank correlation tensors for homogeneous magnetohydrodynamics, Phys. Rev. E 56 (1977) 2875-2888.

[16] J.H. Rubinstein, R. Sinclair, Visualizing Ricci flow of manifolds of revolution, Exp. Math. 14 (2005) 285-298.

[17] M. Schottenloher, A mathematical introduction to conformal field theory. Lectures Notes in Physics. 759 (Springer, Berlin Heiderberg, 2008).

[18] I.N. Vekua, Generalized analytical functions (Nauka, Moscow, 1988). 